\title{
Photochemical Properties of Propargylamine-based Polymers
}

\author{
NATALIA ZAKHAROVA ${ }^{1}$, OLGA SAVINOVA ${ }^{2}$, AKMARAL UTELBAYEVA ${ }^{\text {* }}$ \\ ${ }^{1}$ Sechenov First Moscow State Medical University, Department of Chemistry, 8-2 Trubetskaya Str., 119991 Moscow, Russian Federation \\ ${ }^{2}$ Sechenov First Moscow State Medical University, Department of Pharmacy, 8-2 Trubetskaya Str., 119991 Moscow, Russian Federation \\ ${ }^{3}$ M. Auezov South Kazakhstan State University, Department of Chemistry, 19 A Zheltoksan Str., 160000 Shymkent, Republic of \\ Kazakhstan
}

\begin{abstract}
Propargylamines are a promising but quite poorly studied category of organic compounds. This work aimed to study the photochemical properties of propargylamines polymers containing porphyrin fragments with electron transfer reactions. The study was carried out in 2019 at the Institute of Organic Chemistry (RAS, Moscow, Russia). The obtained polymer was irradiated with a luminous source. The absorption spectra were studied by electron paramagnetic resonance using a radio spectrometer. The presence of porphyrin and propargylamine in the polymer solution has been established to promote the reaction of complexation. This is due to the presence of double bonds in the amino groups, whereas also porphyrin and bromanil, which is an electron acceptor. The resulting data allow explaining the processes involved in photochemical reactions during the irradiation of the porphyrin complex in the presence of bromanil. In the long-wavelength part of the spectrum (above 540 $\mathrm{nm}$ ), electron phototransfer inside the polymer molecule to the porphyrin side and between molecules from the porphyrin anion radical to the neutral polymer molecule take place.
\end{abstract}

Keywords: porphyrines, anions, polymerizations, dynamic processes

\section{Introduction}

Propargylamines represent a promising category of organic compounds within commercial production. These compounds are used in organic synthesis reactions as appropriate monomers [1]. In particular, propargylamines are used in biological synthesis reactions, such as directed synthesis of biologically active substances [2]. Propargylamines are also largely applied for medical purposes. Their activity is known to reduce tumor progression and affect the cardiovascular system by lowering blood pressure values [3]. Furthermore, propargylamines are used to treat Parkinson's disease, as well as to synthesize anti-malaria drugs [4]. One other aspect of the propargylamines practical use has been reported for antidepressants [5]. A wide range of practical applications of propargylamines requires the study of their synthesis reactions and other reactivity capabilities.

Propargylamines have a dual carbon-nitrogen bond, making this category of compounds highly reactive. These bonds allow propargylamines to form a part of complex organic molecules, such as amino acids. Amino acids, on the other hand, are the primary component of proteins, the macromolecules that define the phenomenon of life in earthly conditions [6]. Thus, propargylamines are highly important among organic compounds. At the same time, propargylamines can emit and absorb a certain amount of radiation in the wave spectrum, distinguishing them from most organic compounds. In particular, propargylamines emit photons, and the values of the wave spectrum are strictly individual for each of these compounds [7]. Due to their high reactivity, propargylamines are relatively rare in terrestrial conditions. However, propargylamines are present in sufficiently high concentrations in the gas cloud located at the center of the Milky Way galaxy. In space, these compounds behave in a fairly inert manner in terms of reactivity. This discovery narrowed the potential arrays for extraterrestrial life research [8].

Propargylamines are a universal class of compounds that have been widely used in pharmacology and pharmaceutics in recent years [9]. Some propargylamines derivatives, such as pargyline, rasagiline, and selegiline, are directly used as drugs in the therapy of neurodegenerative disorders, such as Alzheimer's and Parkinson's diseases [10]. Besides, propargylamines are precursors in the organic synthesis of various substrates and are used for the production of natural products [11] and medicines.

\footnotetext{
*email:akmutelbayeva@rambler.ru
} 
Information on the practical application of propargylamines appeared for the first time in the 1940s1950s. Further, these truly unique organic compounds found their application in the synthesis of other aliphatic and aromatic organic compounds and heterocycles, in particular oxazoles, imidazoles, pyrazoles, and quinolines [12]. These compounds are obtained through catalytic reactions involving metals and subsequent cycloaddition [13].

Such popularity of applications is due to the unique structure of propargylamines, which are known to consist of an amino group at the beta-position and an alkyne fragment. Compounds with a triple bond between carbon atoms are characterized by high reactivity when these substances can behave as electrophilic substrates [14]. Besides, they serve as electron sources in nucleophilic reactions [15]. The amine part of the propargylnamine molecule enters into nucleophilic reactions and, subsequently, propargylnamines can be susceptible to chemical transformation reactions. Despite the existence of numerous works, there is no sufficient information on their synthesis and reactivity [16]. In this article, the photochemical properties of propargylamines have been studied. It is known that propargylamines can be used as substrates for the production of many organic compounds of extreme importance in the modern chemical industry [17]. Additionally, propargylamines can be subjected to various additional chemical reactions involving metals as catalysts. As a result, derivatives are formed that can be substrates for the synthesis of organic macromolecules with a more complex structure [18]. Propargylamines, which have the simplest structure, are synthesized by simple alkylation reactions, in particular by amination of propargyl halides, phosphates, or triflates. Among the commercially produced propargylamines (including those used in this study), their derivatives can be alkylated with alkyl halides, as it happens in the synthesis of organic compounds, including such drugs as monoamine oxidase or lysinespecific demethylase inhibitors [19]. Another method for the functionalization of propargylamines is the use of reductive amination reactions. Aldehydes and ketones are used in the presence of NaCNBH3 as a reducing agent [20]. Among other synthesis methods, multicomponent reactions and oxidation reactions of tertiary amines are less popular [21]. In addition, the method of stoichiometric attachment of alkyl nucleophiles to imines or enamines is quite widespread. Alkyl nucleophiles are usually formed by reacting alkynes with metals, such as lithium or magnesium. The reaction requires quite adverse conditions such as the absence of water and low temperatures [22]. Furthermore, over the last decade, the addition of alkynes to imines using transition metals as catalysts has gained popularity among the methods for the synthesis of propargylamines [23]. These methods are justified through the use of a large number of catalytic metals such as copper, zinc, silver, and gold.

As shown in this study, the photochemical properties of propargylamines also represent a promising and great research area since the reaction of electron phototransfer at a certain wavelength specifies a practical application, that is, the use as intermediaries between the conversion of solar energy into electricity.

Conjugated polymers have particular relevance for practical applications. These compounds are unique in that their structural parameters, physico-chemical and electrophysical properties can be chemically adjusted [24]. In this work, an attempt was made to determine how solar energy in polymers with conjugated bonds can be converted into electricity. For this purpose, the mechanisms enabling photosynthesis, i.e., the change under of the compound state to separated charges the influence of light rays, were used. The study of these mechanisms is necessary as it enables the development of other energy sources from renewables, such as solar energy. The considered subject is quite relevant since no previous studies have been done in this direction. Available work on propargylamine is more focused on its synthesis [5, 25-27]. Little attention has been given to the photochemical properties of these compounds, which have determined the relevance of the current research.

This work aimed to examine the photochemical properties of polypropargylamines containing porphyrin fragments with electron transfer reactions. The tasks included: a) to study photochemical reactions involving propargylamine aimed at electron transfer in the presence of quinone, which is an electron acceptor; b) to study the absorption spectrum in the reagent system. 


\section{Materials and methods}

\subsection{Materials}

The study was carried out in 2019 in N. D. Zelinsky Institute of Organic Chemistry (Moscow, Russia, Russian Academy of Sciences). The polymer used in the experiments contains porphyrin and is derived (polymer) from two compounds: propargylamine and phenylacetin. The amino groups of these compounds contain attached deuteroporphyrin, phylloporphyrin, and rhodoporphyrin. The following compounds were used as reagents: proper propargylamine (98\%, manufactured by Fluka, Fluka Chemie Gmbh), methyl alcohol for ultraviolet spectroscopy (chemically pure, grade A, UfaChem, Russia), and chemically pure phenylacetylene (manufactured by Sigma-Aldrich, the USA). Oxalyl chloride (99\%), required for the synthesis of deuteroporphyrin acid chloride was purchased from RT Corporation (Russia, https://reaktivtorg.ru/3375-1/). Chemically pure methylene chloride was purchased there as well (https://reaktivtorg.ru/metilen-khloristyy/).Bromanil (98\%) was purchased from HimReact Corporation, Russia (http://www.himreakt.ru/4215/).

\subsection{Procedures for the synthesis/preparation of a polymeric form of propargylamine}

The synthesis reaction of propargylamine (polymer form, Figure 1) and its co-polymers with phenylacetylene followed by the addition of rhodoporphyrin was carried out according to the schematically illustrated method in Figure 2. The essence of the reaction is to modify the propargylamine polymer with deuteroporphyrin acid chloride, which has been attached to the amino groups of that polymer.

Deuteroporphyrin acid chloride was synthesized by reacting porphyrin with oxalyl chloride. Methylene chloride was used as a reaction medium at standard ambient temperature $\left(+21^{\circ} \mathrm{C}\right)$. In this case, the electron receptor was chemically clean bromanil. Optimal conditions for the synthesis were selected by varying the ratio of the reaction components. The molar ratio of the polymer chain was found to be between 0.12 and 2.00 .

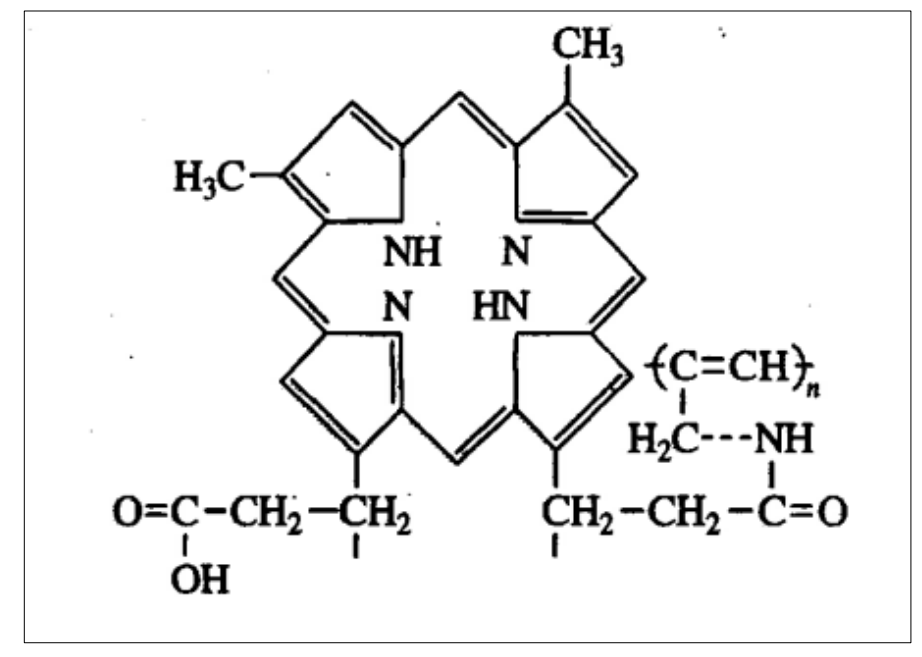

Figure 1. Polypropargylamine polymer with deuteroporphyrin links attached to amino groups

The polymer maximum yield values were $50 \%$ at a minimum ratio of 0.12 . The synthesis products are colored and can also be soluble in polar media. The molecular weight of the synthesis products is $\left(3^{-4}\right) \times 10^{3}$. 


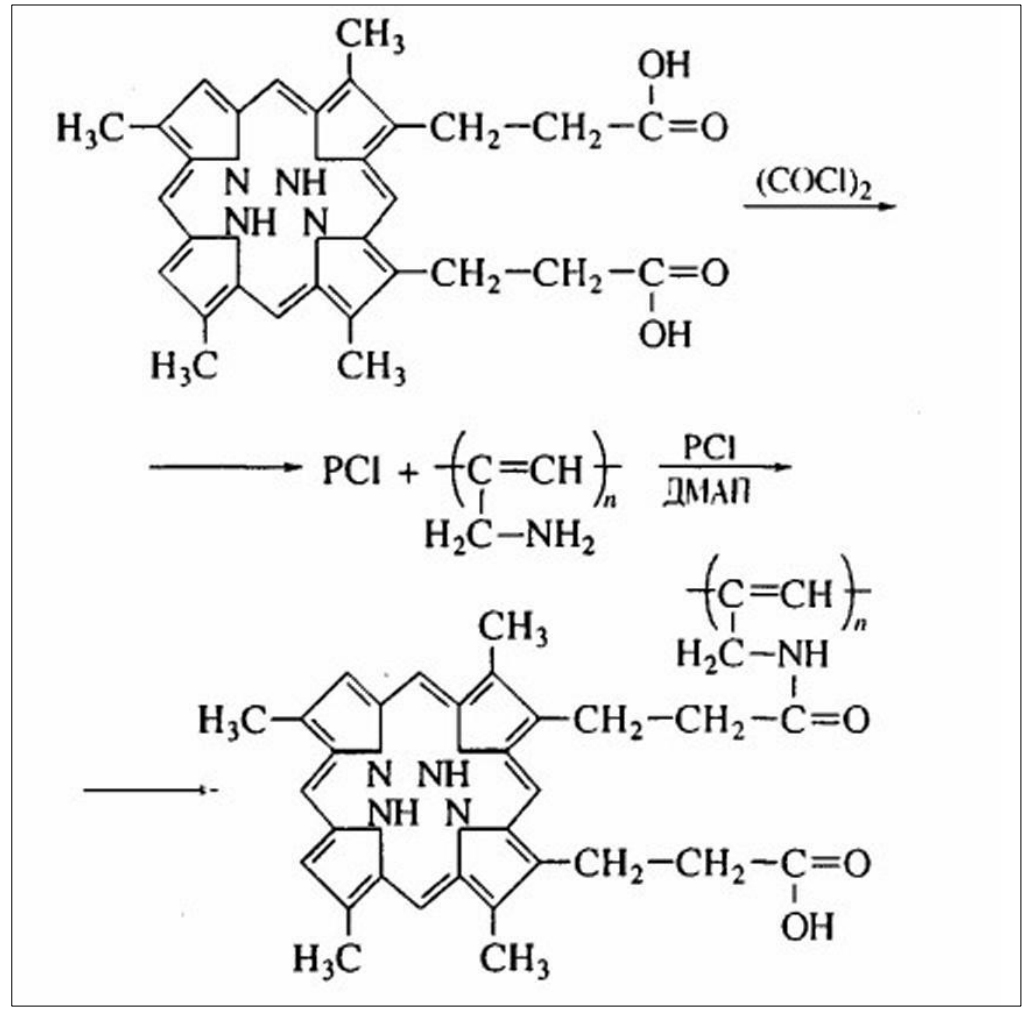

Figure 2. Modification of propargylamine with the attachment of deuteroporphyrin acid chloride.

Note. DMAP stands for dimethylaminopyridine, and P stands for porphyrin

\subsection{The characterizations performed and the equipment used}

The data on the output polymer structure was confirmed by determining the ultraviolet and infrared spectra. The UV spectrum of polyporphyrin represents a superposition of the spectra for two compounds, propargylamine polymer and porphyrin. The values in $\mathrm{nm}$ (nanometers) were 260, 310, 395, 495, 529, 565 , and 620 . For the infrared spectrum, the bands that are characteristic of polyene chains were established. The values in this spectrum were $1600-1610 \mathrm{~cm}^{-1}$ and 3300 . The latter band corresponds to the valence vibrations of N-H chemical bonds. The 1600 band is responsible for the presence of the chemical bond $\mathrm{C}=\mathrm{O}$ in the polymer molecule.

The polymer was irradiated using light from a xenon lamp (modification DKSSh-1000, Russia). The long-wave absorption region within the 540-700 nm range was used, created with a C3C20 light filter (Russia). This lightweight filter has virtually no absorption of the polyene system. By changing the distance between the lamp and the specimen, the intensity of the luminous flux was changed. In this case, the luminous flux intensity indicators were determined using an IMO-2 (Russian) solid-state testometer. The measurement error was within 5\%. Electron paramagnetic resonance spectrum was counted using the RE-1306 (Russia) radio spectrometer at $77 \mathrm{~K}$ using quartz ampoules. Electron absorption spectra were counted using an automatic recording dual-beam spectrophotometer Specord UV-VIS (Germany).

\section{Results and discussions}

Porphyrin and propargylamine in the polymer solution are known to promote the reaction of complex formation. This is due to the presence of conjugated double bonds in the amino groups, fragments of porphyrin, as well as bromanil, which is a pronounced electron acceptor. 


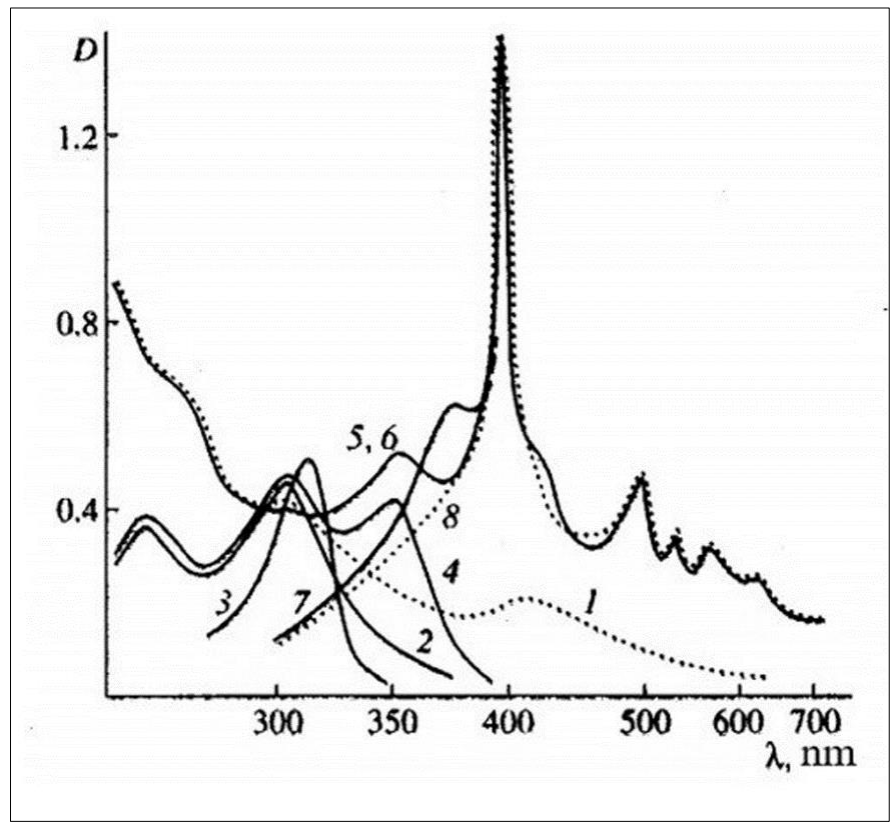

Figure 3. Electronic absorption spectra obtained in methanol solution. 1 - propargylamine polymer, 2 - propargylamine, 3 - bromanil, 4 - porphyrin polymer of propargylamine and bromanil $(5,6)$, 7 - porphyrin and bromanil, 8 - porphyrin. $1-5,8$ - spectra before irradiation, 6,7 - spectra after irradiation with light at a wavelength of $540 \mathrm{~nm}$ and temperature of $77 \mathrm{~K}$

A band at $355 \mathrm{~nm}$ was observed in the absorption spectrum, which can be attributed to complexation when charge transfer between bromanil and polymer amino groups takes place. Figure 3 shows the absorption spectra of the polymer propargylamine, propargylamine, bromanil, and the complex formed by propargylamine and bromanil. The $350 \mathrm{~nm}$ absorption band is visible in spectrum 4 but is absent in spectrum 2, corresponding to propargylamine and in spectrum 3 (bromanil). Thus, the appearance of the band can be explained by the formation of a polymer-bromanil complex when the charge transfers between bromanil and the amino groups of propargylamine. In calculations using mole ratios and taking into account the monomeric link, the complex has been established to have a composition of $1: 1$, and the complexation is in equilibrium at $\mathrm{K}=3.8 \times 102 \mathrm{~L} / \mathrm{mol}$. These data were obtained using the BenesiGildebrand method.

Apparently, the polymer chain apparently does not participate in the reaction of complex formation, which is based on the data where the band location corresponding to the $\pi-\pi^{*}$ transition does not change. This transition takes place in the region of $420 \mathrm{~nm}$ and is associated with the poly-conjugation system. No new bands were detected in the long-wavelength region. Thus, the band in the region of $420 \mathrm{~nm}$ was attributed to absorption, comparing the absorption spectra for propargylamine (spectrum 2, Figure 3 ) and the polymer (spectrum 1, Figure 3 ).

Small values of equilibrium complexation might explain the absence of bands in the absorption spectra like during complexation between bromanil and porphyrin bonds in the polymer composition. Another possible reason is the absorption spectra overlapping of the complex and its components. Similarly, absorption spectra were not recorded in the reaction of complex formation between bromanil and phenyl complexes. This is due to the presence of electron donors with more pronounced properties in the structure of the porphyrin complex.

The irradiation with a wavelength greater than $540 \mathrm{~nm}$ promotes the formation of radical anions of the porphyrin complex. In this case, an electron paramagnetic resonance signal is recorded. Figure 4 shows that the second-order reaction of electron phototransfer is carried out depending on the intensity of the luminous flux. 


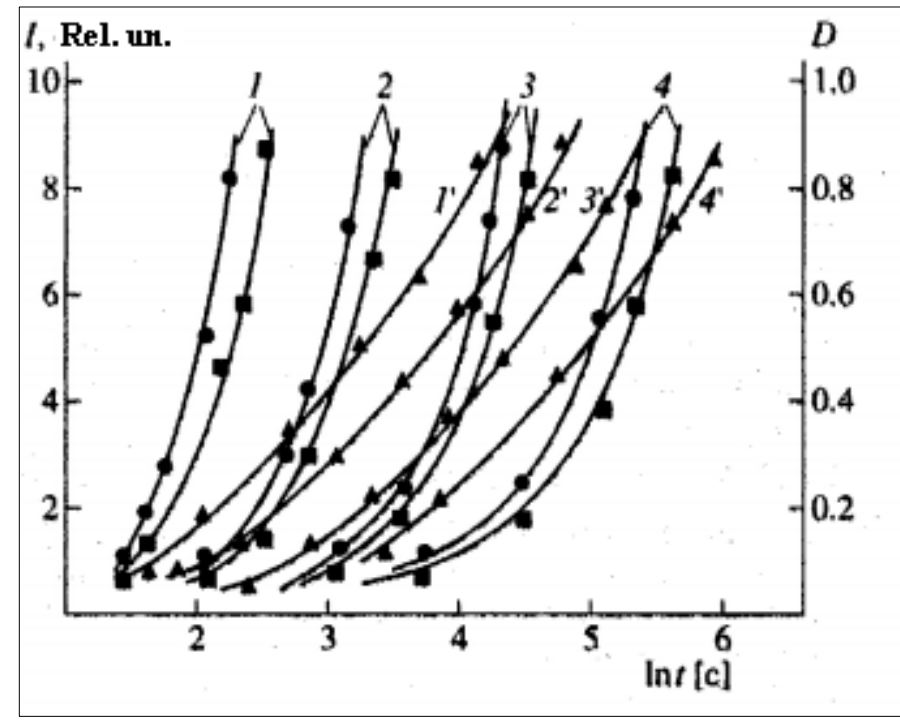

Figure 4. The signal intensity of electron paramagnetic resonance (I axis) and the optical density ( $\mathrm{D}$ axis) of anion radicals as the function of irradiation period $(\mathrm{t})$ by light with a wavelength of $540 \mathrm{~nm}$. Light intensity indices $\mathrm{I} \times 103=6.0(1,1), 5.5(2,2), 3.5(3,3)$, $2.0(4,4) \mathrm{J} / \mathrm{cm}^{2}$. Designations: circles - irradiation of porphyrin polymer, triangles - solution of porphyrin and bromanil, squares - minimum values of the anion-radical yield of porphyrin polymer

Figure 5 shows that the slope value I is equal to 2, which may be a consequence of the two-quantum mechanism of anion radicals formation in the porphyrin parts of the polymer molecule.

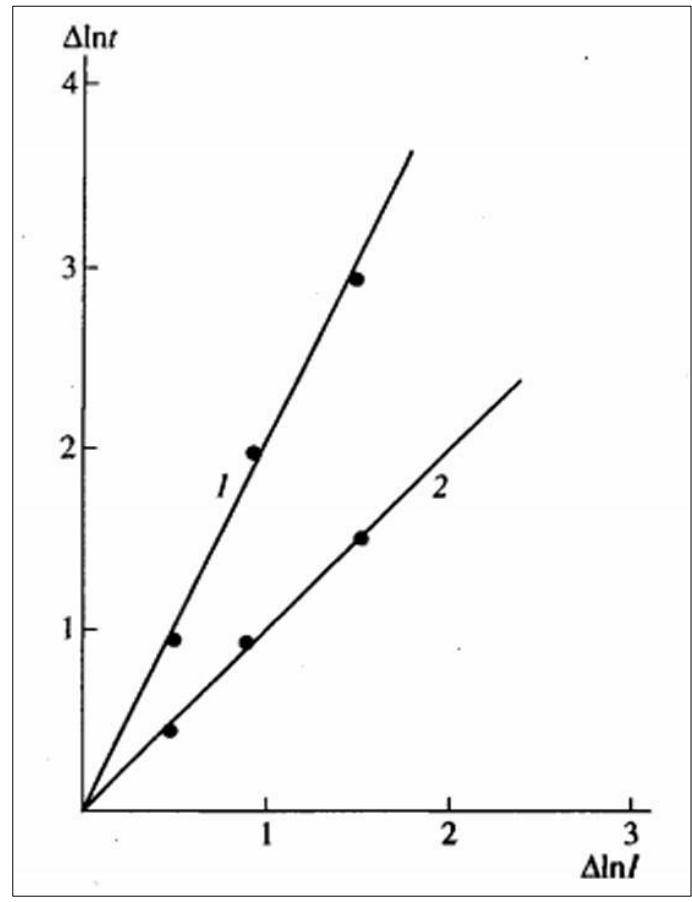

Figure 5. Dependence between the shift of anion-radical accumulation curves and porphyrin polymer (1), bromanil (2), and luminous flux intensity (I)

During irradiation of porphyrin and bromanil solution at a wavelength of more than $540 \mathrm{~nm}$ temperature of $77 \mathrm{~K}$, an ion-radical salt is formed. Therefore, a band in the $382 \mathrm{~nm}$ region is formed in spectrum 7 (Figure 3) as a consequence of the bromanil radical anion formation. Figure 4 also shows the dependence between the optical density of the bromanil absorption band and the irradiation time, taking into account its intensity. Straight line 2 in Figure 5 corresponds to the first order of the reaction in terms of light intensity. That is, the photodeposition of charges occurs according to the $1^{\text {st }}$ quantum principle 
when electron transfers from porphyrin to bromanil.

Bromanil anion radicals are not evident in the absorption spectra when the porphyrin polymer is irradiated in the presence of bromanil. This may indicate a greater probability of intramolecular electron transfer from the polycoupled system to the porphyrin component compared to the other option like electron transfer to bromanil. One minute after switching off the light source, the spectra of porphyrin were not detected anymore. A band was detected in the $382 \mathrm{~nm}$ region, which is a characteristic of the bromanil anion radical.

The data obtained allow explaining the processes of chemical reactions that occur, in particular, during the irradiation of the porphyrin complex in the presence of bromanil. Apparently, the absorption of the first quantum of light inside the molecule involves electron transfer. As a result, a complex is formed, where the porphyrin component is negatively charged, and the propargylamine component is positively charged. Once the second quantum of light is absorbed, an electron phototransfer from the anion to the neutral part, the porphyrin complex, takes place, resulting in the formation of negatively charged porphyrin-polymer molecules in which the porphyrin carries the charge. The acceptor, which is bromanil, takes the electron with the subsequent formation of the anion- radical form. This reaction takes place in the absence of light.

\section{Conclusions}

It has been established that photoexcitation of the porphyrin-propargyl polymer takes place in the presence of bromanil. The following sequence of reactions takes place in the long-wave part of the spectrum (over $540 \mathrm{~nm}$ ): electron phototransfer inside the polymer molecule to the porphyrin part, followed by electron phototransfer between molecules from the porphyrin anion radical to a neutral polymer molecule with the subsequent formation of porphyrin ${ }^{-}$polymer and porphyrin-polymer ${ }^{+}$ions. In the absence of light, the electron transfer reaction from the porphyrin- polymer anion to the bromanil molecule takes place, resulting in the formation of the bromanil-radical anion.

As a result of the reaction, the system is stabilized, with the negative charge located on the bromanil electron acceptor. The positive charge is distributed along the entire length of the polymer chain. Polyconjugated systems involving porphyrin are convenient model systems for the study of reactions corresponding to primary charge separation processes. Polyconjugated systems are, thus, directly involved in charge transfer and photodetachment processes. At the same time, charge stabilization occurs in the polymer macromolecule, in case of charge separation. Undoubtedly, this increases the efficiency of separation of differently charged porphyrin macromolecules.

\section{References}

1.BARANYI, M., PORCEDDU, P.F., GÖLÖNCSÉR, F., KULCSÁR, S., OTROKOCSI, L., KITTEL, Á., PINNA, A., FRAU, L., HULEATT, P.B., KHOO, M.-L., CHAI, C.L.L., DUNKEL, P., MÁTYUS, P., MORELLI, M., SPERLÁGH, B., Novel (Hetero) arylalkenyl propargylamine compounds are protective in toxin-induced models of Parkinson's disease, Molec. Neurodegener., 11(1), 2016, 1-21.

2.ZINDO, F.T., JOUBERT, J., MALAN, S.F., Propargylamine as functional moiety in the design of multifunctional drugs for neurodegenerative disorders: MAO inhibition and beyond. Fut. Medicin. Chem., 7(5), 2015, 609-629.

3.BAR-AM, O., AMIT, T., WEINREB, O., YOUDIM, M.B., MANDEL, S., Propargylamine containing Compounds as modulators of proteolytic cleavage of amyloid protein precursor: involvement of MAPK and PKC activation, J. Alzheimer's Dis., 21(2), 2010, 361-371.

4.BOLEA, I., GELLA, A., UNZETA, M., Propargylamine-derived multitarget-directed ligands: fighting Alzheimer's disease with monoamine oxidase inhibitors. J. Neural Transmiss., 120(6), 2013, 893-902.

5.MAO, F., LI, J., WEI, H., HUANG, L., LI, X., Tacrine-propargylamine derivatives with improved acetylcholinesterase inhibitory activity and lower hepatotoxicity as a potential lead compound for the treatment of Alzheimer's disease, J. Enzyme. Inhib. \& Medic. Chem., 30(6), 2015, 995-1001. 
6.MARCO-CONTELLES, J., UNZETA, M., BOLEA, I., ESTEBAN, G., RAMSAY, R.R., ROMERO, A., MARTÍNEZ-MURILLO, R., CARREIRAS, M.C., ISMAILI, L., ASS234, as a new multi-target directed propargylamine for Alzheimer's disease therapy. Front. Neurosci., 10, 2016, 294.

7.ÖZGERIŞ, B., AKSU, K., TÜMER, F., GÖKSU, S. Synthesis of dopamine, rotigotin, ladostigil, rasagiline analogues 2-amino-4, 5, 6-trimethoxyindane, 1-amino-5, 6, 7-trimethoxyindane, and their sulfamide derivatives. Synthetic Commun., 45(1), 2015, 78-85.

8.BIZZOCCHI, L., PRUDENZANO, D., RIVILLA, V.M., PIETROPOLLI-CHARMET, A., GIULIANO, B.M., CASELLI, P., MARTÍN-PINTADO, J., JIMÉNEZ-SERRA, I., MARTÍN, S., REQUENA-TORRES, M.A., RICO-VILLAS, F., ZENG, S., GUILLEMIN, J.-C. Propargylimine in the laboratory and in space: millimetre-wave spectroscopy and its first detection in the ISM. Astron. \& Astroph., 640, 2020, A98.

9.SCHMITT, M.L., HAUSER, A.T., CARLINO, L., PIPPEL, M., SCHULZ-FINCKE, J., METZGER, E., WILLMANN, D., YIU, T., BARTON, M., SCHÜLE, R., SIPPL, W., JUNG, M., Nonpeptidic propargylamines as inhibitors of lysine specific demethylase 1 (LSD1) with cellular activity, J. Medicin. Chem., 56(18), 2013, 7334-7342.

10.LI, C.J., The development of catalytic nucleophilic additions of terminal alkynes in water, Account Chem. Res., 43(4), 2010, 581-590.

11.PESHKOV, V.A., PERESHIVKO, O.P., VAN DER EYCKEN, E.V., A walk around the A 3coupling, Chem. Society Rev., 41(10), 2012, 3790-3807.

12.CHEN, H., YU, W., GUO, X.H., MENG, W.D., HUANG, Y.G., Facile synthesis of $\alpha$-difluoromethyl a-propargylamines from $\mathrm{CF} 2 \mathrm{H}$-substituted N-tert-butanesulfinyl ketimines. Chinese Chem. Lett., 23(3), 2012, 277-280.

13.QI, R., WANG, X.N., DEKORVER, K.A., TANG, Y., WANG, C.C., LI, Q., LI, H., LV, M.-C., YU, Q., HSUNG, R.P., A convenient synthesis of $\gamma$-amino-ynamides via additions of lithiated ynamides to aryl imines. Observation of an aza-meyer-schuster rearrangement, Synthesis, 45(13), 2013, 1749-1758. 14.XIAO, K.J., WANG, A.E., HUANG, P.Q., Direct transformation of secondary amides into secondary amines: triflic anhydride activated reductive alkylation, Angewandte Chemie, 124(33), 2012, 8439-8442. 15.HUANG, P.Q., HUANG, Y.H., XIAO, K.J., WANG, Y., XIA, X.E., A general method for the onepot reductive functionalization of secondary amides, J. Org. Chemist., 80(5), 2015, 2861-2868.

16.DINDULKAR, S.D., KWAN, B., LIM, K.T., JEONG, Y.T., An eco-sustainable green approach for the synthesis of propargylamines using LiOTf as a reusable catalyst under solvent-free condition, $J$. Chem. Sci., 125(1), 2013, 101-107.

17.XU, H.C., CHOWDHURY, S., ELLMAN, J.A., Asymmetric synthesis of amines using tertbutanesulfinamide., Nat. Prot., 8(11), 2013, 2271-2280.

18.KAUR, P., SHAKYA, G., SUN, H., PAN, Y., LI, G., Chiral N-phosphonyl imine chemistry: an efficient asymmetric synthesis of chiral N-phosphonyl propargylamines, Org. \& Biomolec. Chem., 8(5), 2010, 1091-1096.

198.VERRIER, C., CARRET, S., POISSON, J.F., Asymmetric addition of alkoxy ethynyl anion to chiral N-sulfinyl imines, Org. Lett., 14(19), 2012, 5122-5125.

20.JORDAN, S., STARKS, S.A., WHATLEY, M.F., TURLINGTON, M., Highly stereoselective synthesis of terminal chloro-substituted propargylamines and further functionalization, Org. Lett., 17(19), 2015, 4842-4845.

21.JAEKEL, M., QU, J., SCHNITZER, T., HELMCHEN, G., Addition of organometallic reagents to chiral n-methoxylactams: enantioselective syntheses of pyrrolidines and piperidines, Chemistry-A Europ. J., 19(49), 2013, 16746-16755.

22.YE, L., HE, W., ZHANG, L., A Flexible and Stereoselective Synthesis of Azetidin-3-ones through Gold-Catalyzed Intermolecular Oxidation of Alkynes, Angewandte Chemie, 123(14), 2011, 3294-3297. 23.LAUDER, K., TOSCANI, A., SCALACCI, N., CASTAGNOLO, D., Synthesis and reactivity of propargylamines in organic chemistry, Chem. Rev., 117(24), 2017, 14091-14200. 
24.ARSHADI, S., VESSALLY, E., EDJLALI, L., HOSSEINZADEH-KHANMIRI, R., GHORBANIKALHOR, E.N. Propargylamines: versatile building blocks in the construction of thiazole cores. Beilstein J. Org. Chem., 13(1), 2017, 625-638.

25.HULEATT, P.B., KHOO, M.L., CHUA, Y.Y., TAN, T.W., LIEW, R.S., BALOGH, B., DEME, R., GÖLÖNCSÉR, F., MAGYAR, K., SHEELA, D.P., HO, H.K., SPERLÁGH, B., MÁTYUS, P., CHAI, C.L.L. Novel arylalkenylpropargylamines as neuroprotective, potent, and selective monoamine oxidase B inhibitors for the treatment of Parkinson's disease. J. Medicin. Chem., 58(3), 2015, 1400-1419.

26.TROST, B.M., LI, C.J., Modern alkyne chemistry: Catalytic and atom-economic transformations, Wiley-VCH Verlag GmbH \& Co., Weinheim, 2015.

27.ZHAO, X.B., HA, W., JIANG, K., CHEN, J., YANG, J.L., SHI, Y.P., Efficient synthesis of camptothecin propargylamine derivatives in water catalyzed by macroporous adsorption resin-supported gold nanoparticles, Green Chem., 19(5), 2017, 1399-1406.

Manuscript received: 13. 05. 2021 